excessive pain, indication of purulent inflammation in the affected eye, or if there is irritation, shyness of light, corneal haziness or discoloration of the iris, however slight, in the uninjured eye. The question of a foreign body in an eye renders the probability of an enucleation more probable, but is not per se an indication for the operation.

The question of a poisoned wound one with undoubted contamination with bacteria of any description, demands, as a rule, enucleation. I do not suggest this with the belief in my mind of the migratory theory of sympathetic ophthalmia; on the contrary, I am convinced that the only way for trouble to be started in the second eye is by means of the intimate relations between the lymph channels and spaces, the irides, etc., as shown by the majority of cases exhib. iting a serous uveitis, punctate keratitis, which confirms $m e$ in the belief that a sympathetic ophthalmitis does not arise from a migration of bacteria through the optic nerve. In sympathetic ophthalmia, staphylococci and streptococci are occasionally found in both eyes, but are not the specinic cause of the disease, as shown by our inability to reproduce it by inoculation. It is caused more probably by the irritation of the ciliary nerves producing a reflected disturbance of the blood and nutrition of the uninjured eye.

In panophthalmitis, microbes are plentiful, as one would naturally suppose. Streptococci, staphylococci, and varieties of micrococci, with marked infiltrations of the ciliary body and edema of the papilla.

However, it is not the purpose of this paper to discuss the merits of the different theories of the origin of the trouble; my own belief in the matter is expressed to explain in a measure my position as to the best methods of dealing with or againgt the disease, finding, as I have done, that section of the nerve does not replace enucleation as a preventive measure against sympathetic ophthalmia.

We must bear in mind that this disease is a rare one, and as our operations are mostly done as a prophylactic measure against its onset, or done in cases that have been blind for months or years, it renders our statistics inexact, especially as a sympathetic ophthalmia occasionally sets up after an enucleation has already been done. In this connection I will say that experience has proven that in these cases the inflammation is less virulent than that which occurs when the operation has been too long delayed.

In what cases to enucleate.-Give the operation serious thought in penetrating wounds of the ciliary region, but do not do an operation for every ciliary wound. Enucleate regardless of the situation of the wound in cases where there is marked inflammation of the injured eye and photophobia in the other; also when panophthalmitis is threatened.

The time to enucleate is as soon as possible after deciding it is necessary.

It is seldom that a prophylactic enucleation is followed by a sympathetic ophthalmitis; on the other hand, cures are at times established in cases where the symptoms have been well marked for several weeks. As a rule, however, in case the trouble has commenced, the primarily affected eye should only be removed after the inflammation has been so severe and lasted so long that there can be no hope of restoring vision. If sympathetic ophthalmitis affects the sound eye shortly after an enucleation of the injured, we feel certain that ill-defined and veiled symptoms have been overlooked and it had already begun to suffer before the operation was performed.

Where the inflammation follows after long delay, how can we account for it? As an example note an atrophic globe following an old injury, absolutely quiescent for years, when suddenly an inflammatory action commences, and the fellow eye is lost with sympathetic trouble.

In panophthalmitis an early enucleation should be done for fear of meningitis following. This dreadful sequel has commenced as late as the twentieth day, but generally begins much earlier. We all know and recognize the deformity resulting from the lack of development of the orbit in a child, following enucleation, but this should not deter us from the operation in all suitable cases, for we must feel some pangs of conscience if sympathetic ophthalmitis occurs in a case that we have not operated on for cosmetic reasons. I am strongly inclined to lean toward the operative side when my first examination, shortly after the accident, leads me to feel positive that no sight will be regained in the eye.

\section{WHEN EVISCERATION IS PREFERABLE TO ENUCLEATION.}

Presented to the Section on Ophthalmology, at the Forty-eighth Annual Meeting of the American Medical Association, held at Philadelphia. Pa.. June 1-4, 1897

BY J. C. DUNLAVY, M.D. SIOUX CITY, IOWA.

The services of an eye surgeon are so often demanded in cases of either recent or remote traumatism of the eyeball, as well as for many conditions resulting from disease of this organ, that some general rules of surgical procedure should be adopted for the guidance of those whose advantages for rational information have been necessarily more or less curtailed, and who for lack of proper knowledge are more liable to treat a patient in the generally recognized plan of the surgeons of his community, whether or not this plan is practiced or sanctioned by the better and more enlightened surgeons as being the more rational.

In general surgery we have amputating surgeons, and in eye surgery we have enucleating surgeons, both of whom are perfectly proper and indispensable, but, alas, either are liable to travel at too rapid a pace and leave death and maimed bodies with their baneful results to follow their respective practices along the generations to come.

The surgical procedure of enucleating, or the evisceration of an eyeball, is done for several purposes of more or less seriousness or importance, for a certain class of patients. One class of persons who can not afford to lose extra time from business or labor will often consent to the sacrifice of an eye, when the ball could have been saved entire by proper patience and careful treatment. Others who bear pain poorly will yield rather than undergo the suffering of a panophthalmitis; and finally, the skilful surgeon must demand the surgical means for purely scientific reasons to lessen the danger of the fellow eye. Until comparatively recently it has been almost a wholesale custom of surgeons, more especially those of less scientific attainments, to do the easier, quicker and more brilliant operation of enucleation in all cases calling for such interference, but a few well authenticated cases of death from meningitis following enucle- 
ation during a high stage of inflammation, has aided very materially in calling a halt in this wholesale procedure, and adopting the more conservative and scientific operation of evisceration instead. The rule should be, if any can be established, in an injury to the eyeball, to eviscerate if the injury is of recent occurrence, say within a few days. The theory of this rule is based particularly on the supposition that the migration of septic germs has not yet begun. Whatever the theories of extension of inflammation to the meninges or causes of sympathetic ophthalmitis to the fellow eye, the more rational course to pursue is, for the surgeon to determine from all the conditions of the case, what appears best suited in each individual case. If his judgment be poor, the patient suffers.

It is perfectly rational in this condition, as in all others, to sacrifice as little as possible concurrent with the pathologic state of the eye. I believe no eye surgeon of respectability at the present time would think of enucleating when the globe is filled with pus, for the reason that channels already closed in the optic nerve against migrating septic bodies might, by severing the optic nerve, be opened up and admit the very sources of infection against which nature had fully guarded. And in cases where a foreign body has penetrated the eyeball and resulted in panophthalmitis, and where the foreign body can not be found by evisceration, it is better to enucleate and take the chances of meningitis, but as a rule, avoid everything possible of a traumatic character in either procedure.

In such cases, whatever may have been the cause, the rational procedure is to excise the anterior portion of the globe. The incision should be made far enough back that the iris and ciliary body should be included in that portion of the globe excised; then with a pledget of absorbent cotton dipped into a solution of sublimate, 1 to 2,000, or formol, 1 to 1,000 , thoroughly wipe out all contents of the globe, leaving nothing except a clean and thoroughly washed sclera. This takes time and pains, but it is so rational that it well pays for the trouble. After the globe is thus thoroughly eviscerated and cleaned, a single stitch of No. 3 thread through the conjunctiva. not the sclera, drawn so as to merely shape the outer extremity of the sclera and not close the door completely, and an antiseptic pad of cotton or gauze applied under a light roller completes the operation; after-treatment on the usual plans; this for an eye filled with pus.

In a more recent inflammation, where the eyeball and orbital tissues are very much swollen and a destructive inflammation from some dirty or septic substance having penetrated the interior of the globe, the danger of meningitis is, to my mind, very great, and augmented by any procedure which will in any way tend to irritate the distal extremity of the optic nerve. An eye in this condition should never be enucleated. Eviscerate in practically the same manner as above described, except that the contents of the globe, which are not now purulent, can not be so easily and thoroughly removed by wiping and will need to be removed by the use of some small instrument, such as a scoop, spoon or spatula, introduced between the sclera and what is left of the uveal tract, and the contents in this manner carefully removed. The after-treatment as well as the operation is to be done under strict germicidal rules.

These two pathologic conditions will serve to illus- trate the forms of panophthalmitis where evisceration and not enucleation should be done. In cases where the injury has been of such a nature as to leave cicatrices, which prevent the proper application of an artificial eye or to make the sunken globe appear more unsightly, or a constant dragging or pulling on the orbital tissues, which of itself may excite sympathetic ophthalmia, and from the nature of the injury these conditions are to be expected, the eye should be enucleated. In case of old inflammation, of whatever character, where the ball is tender, sore or subject to subacute attacks, if any part is left remaining the fellow eye is in constant danger and should be protected by the enucleation of the offending eye. In all cases where a foreign body is known to be lodged in the eye and there is greater or less inflammation, if the foreign body is not found by the process of incision or excision of the ball at some point, enucleation should be done.

At least two conditions exist where an eye should not be enucleated: When the globe is filled with pus and during a high state of acute inflammation.

604 Fourth Street.

DISCUSSION.

Dr. H. V. Würdemans of Milwaukee-l rise for the purpose of advocating in the majority of cases a properly applied enucleation. If the operation is completed by sewing up the conjunctiva in the form of a pouch we can get immediate healing and a stump that is partly movable, and if you wish to make the operation a little better you can pick up the recti muscles and bring them out of the wound. The patient can be fitted with an artificial eye within four days to a week afterward. Whoever has had a run of enucleation cases and gone to evisceration and had one case or more of sympathetic irritation will be apt to return to enucleation; whoever has done a number of successful eviscerations and has had one case of meningitis following his enucleation is apt to become a more earnest advocate of evisceration. Eight years ago I brought over a number of glass balls and I may have been unfortunate in having made a failure of the first two cases I operated on. In one I had sympathetic irritation, and since then I have been afraid to return to evisceration. This is despite the paper of Dr. Dunlavy and those of Dr. Fox. I have with me some specimens which I think show the advisability of enucleation [passing specimens]. One of the cases was an enucleation during severe panophthalmitis and the eyeball had to be removed to obtain drainage; simple incision did not give relief to the pus in the orbit.

Dr. J. C. DunlavY-The cosmetic effect has been fully discussed and we will not take it into consideration. Purulent orbital cellulitis should not be considered with this surgical procedure at all, for evisceration could not be expected to evacuate pus in the orbit. Neither enucleation nor evisceration should be adopted by any person as the sole plan of procedure, because there are cases where every man ought to know that one is better than the other. The effort to save as much of the body as possible in the one instance and to lessen the danger of meningitis in the other are the two points I wished to bring out. About two years ago, during a session of the local society, a patient came in with a piece of metal in the eye. I removed it with the magnet and he had no trouble whatever. Some time afterward the patient hurt that same eye and I performed an evisceration, and on wiping out the cavity something caught on the cotton and I found it to be a splinter of metal. I mention it merely because it would be so unlooked for.

The Toxic Principle of Mushrooms.-The active principle of the agaricus muscarius, or fly mushroom, acts by paralysis of the nerve centers, causing death from the heart. There is no doubt that it is one of the most powerful of vegetable poisons. The antidote is the sulphate of atropin, hypodermatically administered at the earliest opportunity. This muscarin in the form of the nitrate or sulphate appears in the laboratory as a brown deliquescent mass, and has a recommendation as an antihydrotic with some virtue as a cure for diabetes insipidus. The eye symptoms in mushroom poisonings appear to be unrecorded. 